\title{
Hecke operators on de Rham cohomology
}

\author{
Min Ho LEE \\ Department of Mathematics, \\ University of Northern lowa, \\ Cedar Falls, IA 50614, U.S.A. \\ lee@math.uni.edu
}

Recibido: 8 de Enero de 2003

Aceptado: 31 de Julio de 2003

\begin{abstract}
We introduce Hecke operators on de Rham cohomology of compact oriented manifolds. When the manifold is a quotient of a Hermitian symmetric domain, we prove that certain types of such operators are compatible with the usual Hecke operators on automorphic forms.
\end{abstract}

Key words: Hecke operators, de Rham cohomology, automorphic forms 2000 Mathematics Subject Classification: 58A12, 11F55

\section{Introduction}

Modular forms, or automorphic forms of one variable, are holomorphic functions on the Poincaré upper half plane $\mathcal{H}$ satisfying a certain transformation formula with respect to an action of a discrete subgroup of $S L(2, \mathbb{R})$. They can be extended to the case of several variables by using holomorphic functions either on the product $\mathcal{H}^{n}$ of $n$ copies of $\mathcal{H}$ for Hilbert modular forms or on the Siegel upper half space $\mathcal{H}_{n}$ of degree $n$ for Siegel modular forms. More general automorphic forms can also be described by using semisimple Lie groups. Indeed, given a semisimple Lie group $G$ of Hermitian type and a discrete subgroup $\Gamma$ of $G$, we can consider automorphic forms for $\Gamma$ defined on the quotient $D=G / K$ of $G$ by a maximal compact subgroup $K$ of $G$. The space $D$ has the structure of a Hermitian symmetric domain, and automorphic forms on $D$ for $\Gamma$ are holomorphic functions on $D$ satisfying a transformation formula for $\Gamma$.

Hecke operators play an important role in number theory, and they act on the space of automorphic forms of various types. Hecke operators acting on other spaces

This research was partially supported by a PDA award from the University of Northern Iowa 
have also been studied in a number of papers over the years. For example, Hecke operators on group cohomology were introduced by Rhie and Whaples [7], and their $q$-analogue was considered in [6]. Such operators were also investigated in [4] and [3] in connection with modular and Hilbert modular forms, respectively. If $f$ is an automorphic form $D$ for $\Gamma$ described above, then $f$ can be interpreted as an algebraic correspondence on the quotient space $\Gamma \backslash D$, which has the structure of a complex manifold assuming that $\Gamma$ is torsion-free. Such a correspondence is determined by a pair of holomorphic maps $\lambda, \mu: \Gamma^{\prime} \backslash D \rightarrow \Gamma \backslash D$, where $\Gamma^{\prime}$ is another discrete subgroup of $G$. The maps $\lambda$ and $\mu$ can be used to construct a Hecke operator on the de Rham cohomology of $\Gamma \backslash D$.

The idea of Hecke operators on cohomology of complex manifolds of the kind described above was suggested, for example, by Kuga and Sampson in [5] (see also [3]). In this paper we extend this idea by introducing Hecke operators on de Rham cohomology of compact oriented manifolds. When the manifold is a quotient of a Hermitian symmetric domain, we prove that special types of such operators are compatible with the usual Hecke operators on automorphic forms.

\section{Hecke operators on cohomology}

In this section we introduce the notion of Hecke operators on de Rham cohomology of a compact oriented manifold. Such an operator is determined by a pair of covering maps of the given manifold.

Let $M$ be a compact oriented manifold of dimension $n$, and let $\mathcal{E}^{r}(M)$ with $0 \leq r \leq$ $n$ denote the space of differential $r$-forms on $M$. If $d^{r}: \mathcal{E}^{r}(M) \rightarrow \mathcal{E}^{r+1}(M)$ denotes the corresponding exterior differentiation map, then the $r$-th de Rham cohomology $M$, which is a vector space over $\mathbb{R}$, is given by

$$
H^{r}(M, \mathbb{R})=\operatorname{Ker} d^{r} / d^{r-1}\left(\mathcal{E}^{r-1}(M)\right) .
$$

Let $M^{\prime}$ be another compact oriented manifold of dimension $n$, and assume that there is a smooth $\ell$-sheeted covering map $\lambda: M^{\prime} \rightarrow M$ for some positive integer $\ell$. Then the associated pull-back map $\lambda^{*}: \mathcal{E}^{r}(M) \rightarrow \mathcal{E}^{r}\left(M^{\prime}\right)$ determines the map

$$
\lambda^{*}: H^{r}(M, \mathbb{R}) \rightarrow H^{r}\left(M^{\prime}, \mathbb{R}\right)
$$

of de Rham cohomology spaces. According to the Poincaré duality, there are isomorphisms

$$
\begin{gathered}
P_{M}: H^{r}(M, \mathbb{R}) \rightarrow H^{n-r}(M, \mathbb{R})^{*}, \\
P_{M^{\prime}}: H^{r}\left(M^{\prime}, \mathbb{R}\right) \rightarrow H^{n-r}\left(M^{\prime}, \mathbb{R}\right)^{*},
\end{gathered}
$$

where $(\cdot)^{*}$ denotes the dual space. Then the Gysin map associated to $\lambda$ is the linear map

$$
\lambda_{*}: H^{r}\left(M^{\prime}, \mathbb{R}\right) \rightarrow H^{r}(M, \mathbb{R})
$$


such that the diagram

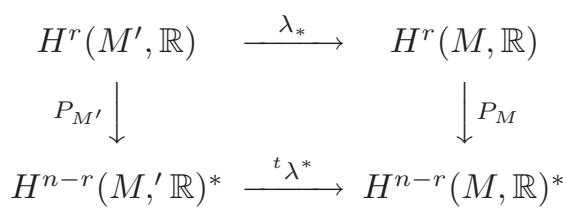

is commutative; here ${ }^{t} \lambda^{*}$ is the dual of the linear map

$$
\lambda^{*}: H^{n-r}(M, \mathbb{R}) \rightarrow H^{n-r}\left(M^{\prime}, \mathbb{R}\right)
$$

(see $[2, \S \mathrm{VI} .4]$ ). Thus the Gysin map is characterized by the condition

$$
\int_{M} \lambda_{*}(\omega) \wedge \xi=\int_{M^{\prime}} \omega \wedge \lambda^{*}(\xi)
$$

for all $\omega \in \mathcal{E}^{r}\left(M^{\prime}\right)$ and $\xi \in \mathcal{E}^{n-r}(M)$. In order to discuss Hecke operators on $H^{r}(M, \mathbb{R})$ we now consider a pair $(\lambda, \mu)$ of smooth $\ell$-sheeted covering maps $\lambda, \mu: M^{\prime} \rightarrow M$.

Definition 2.1. For $0 \leq r \leq n$ the Hecke operator on $H^{r}(M, \mathbb{R})$ associated to the pair $(\lambda, \mu)$ is the map

$$
T^{r}(\lambda, \mu): H^{r}(M, \mathbb{R}) \rightarrow H^{r}(M, \mathbb{R})
$$

given by

$$
T^{r}(\lambda, \mu)=\ell \mu_{*} \circ \lambda^{*},
$$

where $\ell$ is the number of sheets of the covering maps $\lambda$ and $\mu$.

Remark 2.2. If $M$ is a complex manifold, we need to modify the above discussions by considering differential operators with complex coefficients instead of real coefficients, so that the resulting de Rham cohomology will also have coefficients in $\mathbb{C}$. Thus in this case the Hecke operator $T^{r}(\lambda, \mu)$ acts on $H^{r}(M, \mathbb{C})$ and is given by (2), where the covering maps $\lambda$ and $\mu$ are holomorphic.

\section{Quotient spaces}

In this section we discuss some of the properties of the Hecke operators introduced in Section 2 when the manifolds are regarded as quotients of their universal covering space by discrete subgroups of its group of diffeomorphisms.

Let $M$ be a compact oriented manifold of dimension $n$, and let $D$ be its universal covering space. We denote by $\operatorname{Aut}(D)$ the group of diffeomorphisms of $D$, and set

$$
\Gamma=\{\gamma \in \operatorname{Aut}(D) \mid \pi \circ \gamma=\pi\},
$$


where $\pi: D \rightarrow M$ is the covering map. Then $\Gamma$ is a discrete subgroup of $\operatorname{Aut}(D)$ acting on $D$ properly discontinuously, and we can write $M=\Gamma \backslash D$. Thus we see that

$$
\mathcal{E}^{r}(M)=\mathcal{E}^{r}(D)^{\Gamma}
$$

for $0 \leq r \leq n$, where $\mathcal{E}^{r}(D)^{\Gamma}$ denotes the space of $\Gamma$-invariant $r$-forms on $D$.

We now consider a subgroup $\Gamma^{\prime}$ of $\Gamma$ with $\left[\Gamma: \Gamma^{\prime}\right]=\ell$, and set $M^{\prime}=\Gamma^{\prime} \backslash D$. Let $\left\{\varepsilon_{1}, \ldots, \varepsilon_{\ell}\right\}$ be the set of coset representatives of $\Gamma^{\prime}$ in $\Gamma$, so that we have

$$
\Gamma=\coprod_{i=1}^{\ell} \Gamma^{\prime} \varepsilon_{i} .
$$

If $\gamma \in \Gamma$, we shall use the same symbol to denote either the map

$$
\gamma: D \rightarrow D
$$

sending $z \in D$ to $\gamma z \in D$ or the map

$$
\gamma: M^{\prime} \rightarrow M
$$

which it induces.

Theorem 3.1. Let $\lambda, \mu: M^{\prime} \rightarrow M$ be covering maps. Then for $1 \leq r \leq n$ the associated Hecke operator on $H^{r}(M, \mathbb{R})$ is given by

$$
T^{r}(\lambda, \mu) \omega=\sum_{i=1}^{\ell}\left(\lambda \circ \varepsilon_{i}\right)^{*} \omega
$$

for all $\omega \in \mathcal{E}^{r}(D)^{\Gamma}=\mathcal{E}^{r}(M)$.

Proof. First, we shall determine $\mu_{*} \eta$ for $\eta \in \mathcal{E}^{r}\left(M^{\prime}\right)$. By (1) we have

$$
\begin{aligned}
\int_{M^{\prime}} \eta \wedge \mu^{*}(\xi) & =\int_{M} \mu_{*}(\eta) \wedge \xi=\int_{\mu\left(M^{\prime}\right)} \mu_{*} \eta \wedge \xi \\
& =\int_{M^{\prime}} \mu^{*}\left(\mu_{*} \eta \wedge \xi\right)=\int_{M^{\prime}} \mu^{*}\left(\mu_{*} \eta\right) \wedge \mu^{*} \xi
\end{aligned}
$$

for all $\xi \in \mathcal{E}^{n-r}(M)$. Thus $\mu_{*} \eta$ is an element of $\mathcal{E}^{r}(M)$ such that

$$
\mu^{*}\left(\mu_{*} \eta\right)=\eta
$$

Since $\Gamma=\coprod_{i=1}^{\ell} \Gamma^{\prime} \varepsilon_{i}$, for each $\gamma \in \Gamma$ and $1 \leq i \leq \ell$ we have

$$
\Gamma^{\prime} \varepsilon_{i} \gamma=\Gamma^{\prime} \varepsilon_{i_{\gamma}}
$$


for some $i_{\gamma} \in\{1, \ldots, \ell\}$. Thus there is an element $\delta_{i} \in \Gamma^{\prime}$ such that

$$
\varepsilon_{i} \gamma=\delta_{i} \varepsilon_{i_{\gamma}} .
$$

We consider $\eta \in \mathcal{E}^{r}\left(M^{\prime}\right)$ as a $\Gamma^{\prime}$-invariant element of $\mathcal{E}^{r}(D)$, and set $\widetilde{\eta}=\sum_{i=1}^{\ell} \varepsilon_{i}^{*} \eta, \epsilon$ $\mathcal{E}^{r}(D)$. Using (4), we see that

$$
\begin{aligned}
\widetilde{\eta} \circ \gamma & =\sum_{i=1}^{\ell}\left(\eta \circ \varepsilon_{i}\right) \circ \gamma=\sum_{i=1}^{\ell} \eta \circ\left(\varepsilon_{i} \gamma\right)=\sum_{i=1}^{\ell} \eta \circ\left(\delta_{i} \varepsilon_{i_{\gamma}}\right) \\
& =\sum_{i=1}^{\ell}\left(\eta \circ \delta_{i}\right) \circ \varepsilon_{i_{\gamma}}=\sum_{i=1}^{\ell} \eta \circ \varepsilon_{i_{\gamma}}=\sum_{i=1}^{\ell} \eta \circ \varepsilon_{i}=\widetilde{\eta},
\end{aligned}
$$

which shows that $\widetilde{\eta}$ is an element of $\mathcal{E}^{r}(D)^{\Gamma}$. On the other hand, we have

$$
\mu^{*} \widetilde{\eta}(z)=\sum_{i=1}^{\ell} \mu^{*} \varepsilon_{i}^{*} \eta(z)=\sum_{i=1}^{\ell}\left(\varepsilon_{i} \mu\right)^{*} \eta(z)=\sum_{i=1}^{\ell} \eta\left(\varepsilon_{i} \mu z\right)
$$

for all $z \in D$. However, $\mu z$ is simply the $\Gamma$-orbit of $z$, and each $\varepsilon_{i}$ is an element of $\Gamma$; hence we see that $\eta(z)=\eta\left(\varepsilon_{i} \mu z\right)$. Thus we obtain

$$
\mu^{*} \widetilde{\eta}(z)=\sum_{i=1}^{\ell} \eta(z)=\ell \eta(z),
$$

which implies that $\widetilde{\eta} / \ell$ is an element of $\mathcal{E}^{r}(D)^{\Gamma}=\mathcal{E}^{r}(M)$ satisfying $\mu^{*}(\widetilde{\eta} / \ell)=\eta$. By comparing this with (3) we have

$$
\mu_{*} \eta=\frac{1}{\ell} \sum_{i=1}^{\ell} \varepsilon_{i}^{*} \eta
$$

Hence, using (2) and (5), we obtain

$$
T^{r}(\lambda, \mu) \omega=\sum_{i=1}^{\ell} \varepsilon_{i}^{*}\left(\lambda^{*} \omega\right)=\sum_{i=1}^{\ell}\left(\lambda \circ \varepsilon_{i}\right)^{*} \omega
$$

for all $\omega \in \mathcal{E}^{r}(D)^{\Gamma}=\mathcal{E}^{r}(M)$, and therefore the proof of the theorem is complete.

Lemma 3.2. (i) Let $\lambda, \mu: M^{\prime} \rightarrow M$ be covering maps of $M$. Then there is an element $\alpha \in \operatorname{Aut}(D)$ such that $\Gamma^{\prime} \subset \Gamma \cap \alpha^{-1} \Gamma \alpha$.

(ii) If $\alpha \in \operatorname{Aut}(D)$ with $\Gamma^{\prime} \subset \Gamma \cap \alpha^{-1} \Gamma \alpha$, then the map $\alpha: D \rightarrow D$ and the identity map on $D$ induces a pair of covering maps $M^{\prime} \rightarrow M$ of $M$. 
Proof. If $\pi: D \rightarrow M$ is the covering map for the universal covering space $D$ of $M$ as before, there is a unique covering map $\pi_{\mu}: D \rightarrow M^{\prime}$ satisfying $\mu \circ \pi_{\mu}=\pi$. Since both $\pi$ and $\lambda \circ \pi_{\mu}$ are covering maps for the universal covering space $D$ of $M$, there exists $\alpha \in \operatorname{Aut}(D)$ such that

$$
\pi \circ \alpha=\lambda \circ \pi_{\mu}
$$

On the other hand, since $M^{\prime}=\Gamma^{\prime} \backslash D$, for $\gamma^{\prime} \in \Gamma^{\prime}$ we have

$$
\pi\left(\alpha\left(\gamma^{\prime} z\right)\right)=\lambda\left(\pi_{\mu}\left(\gamma^{\prime} z\right)\right)=\lambda\left(\pi_{\mu}(z)\right)=\pi(\alpha(z))
$$

for all $z \in D$. Hence there is an element $\gamma \in \Gamma$ such that $\alpha \gamma^{\prime}=\gamma \alpha$. Thus we have

$$
\alpha \gamma^{\prime} \alpha^{-1}=\gamma \in \Gamma
$$

which implies that $\Gamma^{\prime} \subset \alpha^{-1} \Gamma \alpha$. Since clearly $\Gamma^{\prime} \subset \Gamma$, the proof of (i) is complete. To prove (ii) we take an element $\alpha \in \operatorname{Aut}(D)$ with $\Gamma^{\prime} \subset \Gamma \cap \alpha^{-1} \Gamma \alpha$. If $z_{1}, z_{2} \in D$ represent the same point in $M^{\prime}=\Gamma^{\prime} \backslash D$, then $z_{1}=\gamma^{\prime} z_{2}$ for some $\gamma^{\prime} \in \Gamma^{\prime}$. Since $\alpha \Gamma^{\prime} \alpha^{-1} \subset \alpha \Gamma \alpha^{-1} \cap \Gamma$, we have

$$
\alpha z_{1}=\alpha \gamma^{\prime} z_{2}=\alpha \gamma^{\prime} \alpha^{-1}\left(\alpha z_{2}\right)
$$

with $\alpha \gamma^{\prime} \alpha^{-1} \in \Gamma$; hence $\alpha z_{1}$ and $\alpha z_{2}$ represent the same element in $M$. Thus $\alpha$ : $D \rightarrow D$ induces a covering map $\mu_{\alpha}: M^{\prime} \rightarrow M$. On the other hand, since $\Gamma^{\prime} \subset \Gamma$, the identity map on $D$ determines the other map $\mu_{1}: M^{\prime} \rightarrow M$; hence (ii) follows.

Proposition 3.3. Let $\lambda, \mu: M^{\prime} \rightarrow M$ be as in Lemma 3.2, and let $\pi^{\prime}: M^{\prime \prime} \rightarrow M^{\prime}$ be a smooth covering map with $M^{\prime \prime}=\Gamma^{\prime \prime} \backslash D$, where $\Gamma^{\prime \prime}$ is a subgroup of $\Gamma^{\prime}$ with $\left[\Gamma^{\prime}: \Gamma^{\prime \prime}\right]=r<\infty$. Then we have

$$
T\left(\lambda \circ \pi^{\prime}, \mu \circ \pi^{\prime}\right)=T(\lambda, \mu) .
$$

Proof. Using (2), we have

$$
T\left(\lambda \circ \pi^{\prime}, \mu \circ \pi^{\prime}\right)=\operatorname{lr}\left(\lambda \circ \pi^{\prime}\right)_{*} \circ\left(\mu \circ \pi^{\prime}\right)^{*}=\operatorname{lr} \lambda_{*} \circ\left(\pi_{*}^{\prime} \pi^{\prime *}\right) \circ \mu^{*} .
$$

If $\left\{\delta_{1}, \ldots, \delta_{r}\right\}$ is the set of coset representatives of $\Gamma^{\prime \prime}$ in $\Gamma^{\prime}$, then by (5) we have

$$
\begin{aligned}
\left(\pi_{*}^{\prime} \pi^{\prime *} \omega\right)(z) & =\frac{1}{r} \sum_{i=1}^{r} \delta_{i}^{*}\left(\pi^{\prime *} \omega\right)(z)=\frac{1}{r} \sum_{i=1}^{r}\left(\pi^{\prime} \delta_{i}\right)^{*} \omega(z) \\
& =\frac{1}{r} \sum_{i=1}^{r} \omega\left(\pi^{\prime} \delta_{i} z\right)=\frac{1}{r} \sum_{i=1}^{r} \omega(z)=\omega(z)
\end{aligned}
$$

for $\omega \in \mathcal{E}^{r}\left(M^{\prime}\right)=\mathcal{E}^{r}(D)^{\Gamma}$ and $z \in D$, where we used the fact that $\omega$ is $\Gamma^{\prime}$-invariant. Thus by using this and (2), we see that

$$
T\left(\lambda \circ \pi^{\prime}, \mu \circ \pi^{\prime}\right)=\ell \lambda_{*} \circ \mu^{*}=T(\lambda, \mu) ;
$$

hence the proposition follows. 


\section{Automorphic forms}

In this section we describe automorphic forms on a Hermitian symmetric domain $D$ for a discrete subgroup of the corresponding semisimple Lie group as well as Hecke operators acting on the space of such automorphic forms.

Let $G$ be a semisimple Lie group of Hermitian type, and let $K$ be a maximal compact subgroup of $G$. Then the associated quotient $D=G / K$ has the structure of a Hermitian symmetric domain and can be realized as a bounded domain in $\mathbb{C}^{n}$ for some positive integer $n$ (see e.g. [1], [8]). If $g \in G$ and $z \in D$, we denote by $j(g, z)$ the determinant of the Jacobian matrix at $z$ of the transformation $g: D \rightarrow D, z \mapsto g z$. Thus we have

$$
d(g z)=j(g, z)^{-1} d z
$$

for all $g \in G$, where $d z=d z_{1} \wedge \cdots \wedge d z_{n}$ with $\left(z_{1}, \ldots, z_{n}\right)$ being the standard coordinate system for $\mathbb{C}^{n}$. The resulting map $j: G \times D \rightarrow \mathbb{C}$ is an automorphy factor, which means that it satisfies

$$
j\left(g_{1} g_{2}, z\right)=j\left(g_{1}, g_{2} z\right) j\left(g_{2}, z\right)
$$

for all $z \in D$ and $g_{1}, g_{2} \in G$. Given a function $\phi: D \rightarrow \mathbb{C}$, a nonnegative integer $w$ and an element $g \in G$, we define the function $\left.\phi\right|_{w} g$ on $D$ by

$$
\left(\left.\phi\right|_{w} g\right)(z)=j(g, z)^{w} \phi(g z)
$$

for all $z \in D$.

Lemma 4.1. Given $\phi: D \rightarrow \mathbb{C}$ and a nonnegative integer $w$, we have

$$
\left.\left(\left.\phi\right|_{w} g\right)\right|_{w} g^{\prime}=\left.\phi\right|_{w}\left(g g^{\prime}\right)
$$

for all $g, g^{\prime} \in G$.

Proof. Given $g, g^{\prime} \in G$, we have

$$
\begin{aligned}
\left(\left.\left(\left.\phi\right|_{w} g\right)\right|_{w} g^{\prime}\right)(z) & =j\left(g^{\prime}, z\right)^{w}\left(\left.\phi\right|_{w} g\right)\left(g^{\prime} z\right) \\
& =j\left(g^{\prime}, z\right)^{w} j\left(g, g^{\prime} z\right)^{w} \phi\left(g g^{\prime} z\right) \\
& =j\left(g g^{\prime}, z\right)^{w} \phi\left(g g^{\prime} z\right) \\
& =\left(\left.\phi\right|_{w}\left(g g^{\prime}\right)\right)(z)
\end{aligned}
$$

for all $z \in D$, where we used (7).

We now consider a discrete subgroup $\Gamma$ of $G$ acting on $D$ properly discontinuously.

Definition 4.2. A holomorphic function $\phi: D \rightarrow \mathbb{C}$ is an automorphic form of weight $w$ for $\Gamma$ if it satisfies

$$
\left.\phi\right|_{w} \gamma=\phi
$$

for all $\gamma \in \Gamma$. We denote by $\mathcal{M}_{w}(\Gamma)$ the complex vector space consisting of all automorphic forms of weight $w$ for $\Gamma$. 
Example 4.3. Let $G$ be the symplectic group $S p(m, \mathbb{R})$ of degree $m$. Then the associated Hermitian symmetric domain can be regarded as the Siegel upper half space $\mathcal{H}_{m}$ of degree $m$, which consists of the complex symmetric $m \times m$ matrices with positive definite imaginary part. An element $g=\left(\begin{array}{ll}a & b \\ c & d\end{array}\right) \in S p(m, \mathbb{R})$ acts on $\mathcal{H}_{m}$ by

$$
g z=(a z+b)(c z+d)^{-1}
$$

for all $z \in \mathcal{H}_{m}$, and the corresponding automorphy factor is given by

$$
j(g, z)=\operatorname{det}(c z+d)^{-(m+1)} .
$$

Let $\Gamma$ be a discrete subgroup of $S p(m, \mathbb{R})$. Then an automorphic form on $\mathcal{H}_{m}$ of weight $w$ for $\Gamma$ is a holomorphic function $\phi: \mathcal{H}_{m} \rightarrow \mathbb{C}$ satisfying

$$
\phi(\gamma z)=\operatorname{det}(c z+d)^{(m+1) w} \phi(z)
$$

for all $z \in \mathcal{H}_{m}$ and $\gamma=\left(\begin{array}{ll}a & b \\ c & d\end{array}\right) \in \Gamma$. Such a function is known as a Siegel modular form of weight $(m+1) w$ for $\Gamma$.

Given $\alpha \in G$, we denote by $\Gamma_{(\alpha)}$ the subgroup of $\Gamma$ given by

$$
\Gamma_{(\alpha)}=\Gamma \cap \alpha^{-1} \Gamma \alpha .
$$

We assume that $\left[\Gamma: \Gamma_{(\alpha)}\right]<\infty$ and that

$$
\Gamma=\coprod_{i=1}^{s} \Gamma_{(\alpha)} \delta_{i}
$$

with $\delta_{1}, \ldots, \delta_{s} \in \Gamma$. Given $\phi: D \rightarrow \mathbb{C}$ and a nonnegative integer $w$, we define the function $T_{w}(\alpha) \phi: D \rightarrow \mathbb{C}$ by

$$
T_{w}(\alpha) \phi=\left.\sum_{i=1}^{s} \phi\right|_{w} \delta_{i} .
$$

Lemma 4.4. Given $\alpha \in G$ satisfying (10), the formula (11) determines a complex linear map $T_{w}(\alpha): \mathcal{M}_{w}(\Gamma) \rightarrow \mathcal{M}_{w}(\Gamma)$.

Proof. Since the map $\phi \mapsto T_{w}(\alpha) \phi$ is clearly linear, it suffices to show that

$$
T_{w}(\alpha)\left(\mathcal{M}_{w}(\Gamma)\right) \subset \mathcal{M}_{w}(\Gamma) .
$$

Using Lemma 4.1, for each $\gamma \in \Gamma$ and $\phi \in \mathcal{M}_{w}(\Gamma)$ we see that

$$
\begin{aligned}
\left.\left(T_{w}(\alpha) \phi\right)\right|_{w} \gamma & =\left.\sum_{i=1}^{s}\left(\left.\phi\right|_{w} \delta_{i}\right)\right|_{w} \gamma \\
& =\left.\sum_{i=1}^{s} \phi\right|_{w}\left(\delta_{i} \gamma\right)=T_{w}(\alpha) \phi,
\end{aligned}
$$

where we used the fact that $\left\{\delta_{1} \gamma, \ldots, \delta_{u} \gamma\right\}$ is a set of coset representatives of $\Gamma_{(\alpha)}$ in $\Gamma$. Hence we see that $T_{w}(\alpha) \phi \in \mathcal{M}_{w}(\Gamma)$, and therefore the lemma follows. 
Definition 4.5. The complex linear map $T_{w}(\alpha): \mathcal{M}_{w}(\Gamma) \rightarrow \mathcal{M}_{w}(\Gamma)$ in Lemma 4.4 is the Hecke operator on $\mathcal{M}_{w}(\Gamma)$ determined by $\alpha$.

\section{Automorphic forms and cohomology}

In this section we establish a correspondence between the space of modular forms of weight one and a subspace of the de Rham cohomology space and prove that the Hecke operators on modular forms are compatible with a special type of Hecke operators on de Rham cohomology under this correspondence.

Let $G$ and $D$ be as in Section 4, and let $\Gamma$ be a torsion-free discrete subgroup of $G$. Then the quotient space $X=\Gamma \backslash D$ has the structure of a complex manifold of dimension $n$. We assume that $X$ is compact. Then for each $r \in\{0,1, \ldots, n\}$ the $r$-th de Rham cohomology $H^{r}(X, \mathbb{C})$ of $X$ with coefficients in $\mathbb{C}$ has a Hodge decomposition of the form

$$
H^{r}(X, \mathbb{C})=\sum_{p+q=r} H^{p, q}(X),
$$

where the $H^{p, q}(X)$ are Dolbeault cohomology groups with

$$
\overline{H^{p, q}(X)}=H^{q, p}(X) .
$$

Given $p, q \in \mathbb{Z}$ with $0 \leq p, q \leq r$ and $p+q=r$, we denote by

$$
\varpi_{p, q}: H^{r}(X, \mathbb{C}) \rightarrow H^{p, q}(X)
$$

the natural projection map. If $\Omega^{p}(X)$ denotes the sheaf of holomorphic $p$-forms on $X$, then there is a canonical isomorphism

$$
H^{p, q}(X)=H^{q}\left(X, \Omega^{p}(X)\right) .
$$

Lemma 5.1. There is a canonical isomorphism

$$
\mathcal{M}_{1}(\Gamma) \cong H^{0, n}(X)
$$

where $n$ is the dimension of $X$.

Proof. We identify $H^{0, n}(X)$ with $H^{0}\left(X, \Omega^{n}(X)\right)$, so that each element of $H^{0, n}(X)$ can be regarded as a $\Gamma$-invariant $n$-form

$$
\omega(z)=f(z) d z=f(z) d z_{1} \wedge \cdots \wedge d z_{n} \in \Omega^{n}(D)
$$

on $D$, where $z=\left(z_{1}, \ldots, z_{n}\right)$ is the standard coordinate system for $D \subset \mathbb{C}^{n}$. For such $\omega$ we set

$$
\mathcal{L}(\omega)=f
$$


Given $\gamma \in \Gamma$, using (6) and (8), we have

$$
\omega(\gamma z)=f(\gamma z) d(\gamma z)=f(\gamma z) j(\gamma, z)^{-1} d z=\left(\left.f\right|_{1} \gamma\right)(z) d z .
$$

Since $\omega$ is $\Gamma$-invariant, we see that

$$
\left.\mathcal{L}(\omega)\right|_{1} \gamma=\left.f\right|_{1} \gamma=f=\mathcal{L}(\omega)
$$

hence we have $\mathcal{L}(\omega) \in \mathcal{M}_{1}(\Gamma)$. On the other hand, given $h \in \mathcal{M}_{1}(\Gamma)$, we set

$$
\omega_{h}(z)=h(z) d z \in \Omega^{n}(D) .
$$

Using the fact that $h$ is an automorphic form of weight one, we have

$$
\omega_{h}(\gamma z)=h(\gamma z) d(\gamma z)=h(\gamma z) j(\gamma, z)^{-1} d z=h(z) d z=\omega_{h}(z) .
$$

Hence $\omega_{h}$ is $\Gamma$-invariant and therefore is an element of $H^{0, n}(X)$. Clearly, we have

$$
\mathcal{L}\left(\omega_{h}\right)=h
$$

hence the proof of the lemma is complete.

Let $\alpha \in G$ with $\left[\Gamma: \Gamma_{(\alpha)}\right]<\infty$, where $\Gamma_{(\alpha)}$ is as in (9). If $\gamma^{\prime} \in \Gamma_{(\alpha)}$, then $\alpha \gamma^{\prime} \alpha^{-1} \in \Gamma$; hence for each $z \in D$ we have

$$
\alpha\left(\gamma^{\prime} z\right)=\left(\alpha \gamma^{\prime} \alpha^{-1}\right) \alpha z \in \Gamma(\alpha z) .
$$

Thus it follows that $\alpha\left(\Gamma_{(\alpha)} z\right) \subset \Gamma(\alpha z)$, and therefore the map $\alpha: D \rightarrow D, z \mapsto \alpha z$ induces a map $\mu_{\alpha}: X_{(\alpha)} \rightarrow X$, where $X_{(\alpha)}=\Gamma_{(\alpha)} \backslash D$. On the other hand, since $\Gamma_{(\alpha)} \subset \Gamma$, there is another map $\mu_{1}: X_{(\alpha)} \rightarrow X$ induced by the identity map on $D$. Thus the maps $\mu_{1}$ and $\mu_{\alpha}$ determine the Hecke operator $T^{r}\left(\mu_{1}, \mu_{\alpha}\right)$ acting on the $r$-th de Rham cohomology space $H^{r}(X, \mathbb{C})$ of $X$ with coefficients in $\mathbb{C}$ (see Remark 2.2). We denote by

$$
\Xi: H^{n}(X, \mathbb{C}) \rightarrow \mathcal{M}_{1}(\Gamma)
$$

the map determined by the projection map $(12)$ with $(p, q)=(0, n)$ and the canonical isomorphism (13). Thus, if $\mathcal{L}$ is as in (14), we have

$$
\Xi=\mathcal{L} \circ \varpi_{0, n}
$$

Now we are ready to state our main theorem in this section, which states that the Hecke operators $T\left(\mu_{1}, \mu_{\alpha}\right)$ and $T(\alpha)$ are compatible.

Theorem 5.2. Let $\alpha \in G$ with $\Gamma=\coprod_{i=1}^{s} \Gamma_{(\alpha)} \delta_{i}$ for some $\delta_{1}, \ldots, \delta_{s} \in \Gamma$. Then the diagram

is commutative.

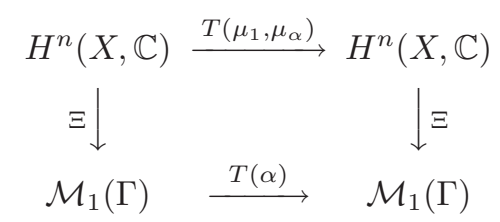


Proof. Given nonnegative integers $p$ and $q$ with $p+q=n$, we consider the sets $A_{p}$ and $B_{q}$ of multi-indices given by

$$
\begin{aligned}
& A_{p}=\left\{\xi=\left(\xi_{1}, \ldots, \xi_{p}\right) \mid 1 \leq \xi_{1}<\cdots<\xi_{p} \leq n\right\}, \\
& B_{q}=\left\{\eta=\left(\eta_{1}, \ldots, \eta_{q}\right) \mid 1 \leq \eta_{1}<\cdots<\eta_{q} \leq n\right\} .
\end{aligned}
$$

Each element $\omega$ of the $n$-th de Rham cohomology group $H^{n}(X, \mathbb{C})$ of $X$ can be regarded as a $\Gamma$-invariant $n$-form of the type

$$
\omega(z)=\sum_{p+q=n} \sum_{(\xi, \eta) \in A_{p} \times B_{q}} \phi_{(\xi, \eta)}(z) d z_{\xi} \wedge d \bar{z}_{\eta}
$$

where $d z_{\xi}=d z_{\xi_{1}} \wedge \cdots \wedge d z_{\xi_{p}}$ and $d \bar{z}_{\eta}=d \bar{z}_{\eta_{1}} \wedge \cdots \wedge d \bar{z}_{\eta_{q}}$. Using (15), we have

$$
(\Xi \omega)(z)=\left(\mathcal{L} \circ \varpi_{0, n}\right) \omega(z)=\mathcal{L}\left(\phi_{\varepsilon}(z) d z_{1} \wedge \cdots \wedge d z_{n}\right)=\phi_{\varepsilon}(z) \in \mathcal{M}_{1}(\Gamma),
$$

where $\varepsilon=(1, \ldots, n)$. Thus by (11) we see that

$$
T(\alpha) \Xi(\omega)=\left.\sum_{i=1}^{s} \phi_{\varepsilon}\right|_{1} \delta_{i} .
$$

On the other hand, using (2), (5) and the fact that $\mu_{1}, \mu_{\alpha}: X_{(\alpha)} \rightarrow X$ are $s$-sheeted covering maps, we have

$$
T\left(\mu_{1}, \mu_{\alpha}\right) \omega=s\left(\mu_{\alpha_{*}} \circ \mu_{1}^{*}\right) \omega=s \mu_{\alpha_{*}} \omega=\sum_{i=1}^{s} \delta_{i}^{*} \omega .
$$

Thus we obtain

$$
\begin{aligned}
\left(\left(\Xi \circ T\left(\mu_{1}, \mu_{\alpha}\right)\right) \omega\right)(z) & =\sum_{i=1}^{s}\left(\mathcal{L} \circ \varpi_{0, n}\right)\left(\phi_{\varepsilon}\left(\omega\left(\delta_{i} z\right)\right)\right. \\
& =\sum_{i=1}^{s} \mathcal{L}\left(\phi_{\varepsilon}\left(\delta_{i} z\right) d\left(\delta_{i} z\right)\right) \\
& =\sum_{i=1}^{s} \mathcal{L}\left(\left(\left.\phi_{\varepsilon}\right|_{1} \delta_{i}\right)(z) d z\right)=\sum_{i=1}^{s}\left(\left.\phi_{\varepsilon}\right|_{1} \delta_{i}\right)(z) .
\end{aligned}
$$

Hence the theorem follows from this and (16).

\section{References}

[1] W. L. Baily Jr., Introductory lectures on automorphic forms, Iwanami Shoten, Publishers, Tokyo, 1973 . 
[2] M. Karoubi and C. Leruste, Algebraic topology via differential geometry, London Mathematical Society Lecture Note Series, vol. 99, Cambridge University Press, Cambridge, 1987, ISBN 0521-31714-2.

[3] M. Kuga, Group cohomology and Hecke operators. II. Hilbert modular surface case, Automorphic Forms and Number Theory (Sendai, 1983), Adv. Stud. Pure Math., vol. 7, North-Holland, Amsterdam, 1985, pp. 113-148.

[4] M. Kuga, W. Parry, and C. H. Sah, Group cohomology and Hecke operators, Manifolds and Lie Groups (Notre Dame, Ind., 1980), Progr. Math., vol. 14, Birkhäuser Boston, Mass., 1981, pp. 223-266.

[5] M. Kuga and J. H. Sampson, A coincidence formula for locally symmetric spaces, Amer. J. Math. 94 (1972), 486-500.

[6] M. H. Lee, Hecke operators on the q-analogue of group cohomology, Beiträge Algebra Geom. 42 (2001), 59-69.

[7] Y. H. Rhie and G. Whaples, Hecke operators in cohomology of groups, J. Math. Soc. Japan 22 (1970), 431-442.

[8] I. Satake, Algebraic structures of symmetric domains, Kanô Memorial Lectures, vol. 4, Iwanami Shoten, Tokyo, 1980. 